\title{
Medical Rice as a Whole Grain: A New Technological Advancement By Shaw Watanabe, For Prevention of Cardiometabolic Diseases and Dementia
}

\author{
Ram B Singh ${ }^{1}$, Shaw Watanabe ${ }^{2}$, Viliam Mojto*3 ${ }^{*}$ Toru Takahashi ${ }^{4}$, Rie Horuichi ${ }^{4}$, Kumar Kartikey $^{5}$ and \\ Kolcunova Martina ${ }^{3}$ \\ ${ }^{1}$ Halberg Hospital and Research Institute, India \\ ${ }^{2}$ Clinical \& Functional Nutriology, Life Science, Japan
}

${ }^{3} 1^{\text {st }}$ Internal Clinic Faculty of Medicine Comenius University Bratislava, Slovakia

${ }^{4}$ Fukuoka University, Japan

${ }^{5}$ Halberg Hospital and Research Institute,Moradabad,India

*Corresponding author: Viliam Mojto, $3^{\text {rd }}$ Internal Clinic, Faculty of Medicine,Comenius University, Bratislava, Slovakia

\begin{tabular}{|c|c|}
\hline ARTICLE INFO & ABSTRACT \\
\hline Received: 崑 February 02, 2019 & Abbreviations: CMD: Cardiometabolic Disease; MMSE: Mini Mental State Examination; \\
\hline Published: 幽 February 19, 2019 & $\begin{array}{l}\text { CDT: Clock Drawing Test; DASH: Dietary Approaches to Stop Hypertension; GABA: Gamma } \\
\text { Aminobutyric Acid }\end{array}$ \\
\hline
\end{tabular}

Citation: Ram B Singh, Shaw Watanabe, Viliam Mojto, Toru Takahashi, Rie Horuichi, Kumar Kartikey. Medical Rice as a Whole Grain: A New Technological Advancement By Shaw Watanabe, For Prevention of Cardiometabolic Diseases and Dementia. Biomed J Sci \& Tech Res 14(4)-2019. BJSTR. MS.ID.002595.

\section{Introduction}

\section{Background}

Dementia has become a public health problem due to its impact on health and daily life. Major risk factors of dementia are cardiometabolic disease(CMDs), tobacco and lack of physical exercise. Western diet is an emerging risk factor of dementia due to its influence on all these risk factors of CMDs. However, Western diet may be an independent risk factor of dementia due its lack of antioxidant vitamins, and flavonoids, fiber, omega-3 fatty acids and essential amino acids in these diets. The Lyon Heart study, The Indo-Mediterranean Diet Heart study and the PREDIMED study showed that Mediterranean style diets that are rich in whole grains can protect against CMDs and cancer [1-4]. These diets are also known to decrease psychological disorders and dementia due to high content of flavonoids [5-8]. This review aims to examine, if Mediterranean style diets, in particular whole grains, including medical rice can inhibit cognitive decline and dementia.

\section{Mediterranean Style Diets and Risk of Dementia}

Mediterranean style diets are rich in fruits, vegetables, whole grains, nuts, olive oil and fish with lower red meat and preserved meats [4]. These diets can lower the risk of cognitive decline by reducing the risk of developing CMDs, a known risk factor for dementia, as well as by their direct effect [4-8]. The mechanism of action of this diet is that it also has an anti-oxidant and antiinflammatory effects that are major mechanisms underlying dementia [4] found that there is strong evidence for protective effects of vitamin E, B vitamins, magnesium, vitamin D, flavonoids, 
amino acids and n-3 fatty acids, and deleterious effects of sugary and rapidly absorbed refined fast foods, saturated and trans fat, red and preserved meats on dementia [1,3,9]. Among specific foods with evidence of neuroprotection are leafy and other vegetables, berries, whole grains (beans and pulses, porridge, grams, flex seeds, seeds, soya beans and millets) and seafood [4-9]. The PREDIMED study, assessed 522 participants at high vascular risk (44.6\% men, age $74.6 \pm 5.7$ years at cognitive evaluation) enrolled in a multicentre, randomised, primary prevention trial [7]. After adjustment for sex, age, education, Apolipoprotein E genotype, family history of cognitive impairment/dementia, smoking, physical activity, body mass index, hypertension, dyslipidaemia, diabetes, alcohol and total energy intake, participants allocated to the Meddiet + EVOO showed higher mean mini mental state examination (MMSE) and clock drawing test (CDT) scores with significant differences versus control (adjusted differences: $+0.6295 \% \mathrm{CI}+0.18$ to +1.05 , $\mathrm{p}=0.005$ for MMSE, and $+0.5195 \% \mathrm{CI}+0.20$ to $+0.82, \mathrm{p}=0.001$ for CDT).

The adjusted means of MMSE and CDT scores were also higher for participants allocated to the Meddiet+Nuts versus control (adjusted differences: +0.57 (95\% CI +0.11 to +1.03 ), $\mathrm{p}=0.015$ for MMSE and +0.33 (95\% CI +0.003 to +0.67$), p=0.048$ for CDT). It is possible that an intervention with Meddiets enhanced with either EVOO or nuts appears to improve cognition compared with a low-fat diet [7]. In a meta-analysis 32 studies from 25 unique cohorts, including 5 randomized, controlled trials and 27 observational studies, met the inclusion criteria [5]. The majority of studies showed that the Mediterranean style diet was associated with improved cognitive function, a decreased risk of cognitive impairment or decreased risk of dementia, or Alzeimers disease. Of the total, 3 studies reported no correlation between the diet and Alzheimer's disease, another 3 studies reported no association between the diet and cognitive impairment, and 5 studies found no association between the diet and cognitive function. In view of the large heterogeneity, differences in quality and the limitations in study design, it is possible that adherence to the Mediterranean style diet was associated with better cognitive performance [5] In a more recent review, following PRISMA guidelines and was conducted using four databases and resulted in 31 articles of interest [6]. Cross-sectional studies and cohort studies in the nonMediterranean region showed mixed results.

However, cohort studies in the Mediterranean region and randomized controlled trials showed more cohesive outcomes of the beneficial effect of the Mediterranean style diets on cognitive function [6]. Although more standardized and in-depth studies are needed to strengthen the existing body of evidence, results from this review indicate that the Mediterranean diet may have a major role in cognitive health and risk of Alzheimer's disease and dementia. According to another review, several studies have examined dietary patterns, particularly the Mediterranean and DASH (Dietary Approaches to Stop Hypertension) diets. However, neither of these diets are tailored to the specific foods and nutrients that have been identified as neuroprotective [9]. A newly developed diet, called MIND (Mediterranean-DASH Intervention for Neurodegenerative Delay), incorporates many elements of the Mediterranean and DASH diets but with modifications that reflect the best evidence for neuronal protection. These problems of the association of Mediterranean style diets with dementia may be explained by inadequate attention to nutrient/food intake in the interpretation of results of the study. In a further study, the German Study on Aging, Cognition and Dementia in Primary Care Patients (AgeCoDe), dietary intake of red wine, white wine, coffee, green tea, olive oil, fresh fish, fruits and vegetables, red meat and sausages, was assessed by a single-food-questionnaire [10]. Participants aged 75+ of cohort $(n=2622)$ were regularly followed over 10 years for incidence of Alzheimer's disease $(n=418)$. The results showed that only higher red wine intake was associated with a lower incidence of Alzheimer's disease ( $\mathrm{HR}=0.92 ; \mathrm{P}=0.045)$. However, this was true only for men ( $\mathrm{HR}=0.82 ; \mathrm{P}<0.001)$, while in women higher red wine intake was associated with a higher incidence of Alzheimer's disease (HR $=1.15 ; \mathrm{P}=0.044)$, and higher white wine intake with a more pronounced memory decline over time ( $\mathrm{HR}=-0.13$; $\mathrm{P}=0.052)$ [10]. It is clear that no evidence was observed for these single foods to be protective against cognitive decline, with the exception of red wine, which reduced the risk for Alzheimer's disease only in men.

\section{Effects of Flavonoids on Dementia}

It is possible that the most effective dietary ingredient responsible for beneficial effects of foods on dementia may be flavonoids which are also rich in whole grains such as Medical rice, legumes, millets, flex seeds, corn, etc $[11,12]$. Flavonoids constitute a large group of polyphenolic compounds with numerous beneficial effects on behavior and cognition, which vary from learning and memory enhancement to an improvement of general cognition. Flavonoids have also been implicated in neuronal proliferation and survival, by acting on a variety of cellular signaling cascades, including the ERK/CREB/BDNF and PI3K/Akt pathway, reduction of oxidative stress and relief from clinical manifestations of Alzheimer's disease $[8,11]$. Electrophysiological aspect indicate that flavonoids can promote long term potentiation in the hippocampus, supporting the hypothesis of synaptic plasticity mediation indicating a neuroprotective effect of flavonoid compounds in the brain. It is possible that flavonoid content of Mediterranean foods could be a potential clinical direction for prevention and/or attenuation of cognitive decline deterioration which accompanies various brain disorders [11-20] \& (Table 1). A recent review included 32 studies from 25 unique cohorts, including 5 randomized, controlled trials and 27 observational studies [21-27]. The majority of studies showed that the Mediterranean style diets was associated with improved cognitive function, a decreased risk of cognitive impairment or decreased risk of dementia. Three studies found no correlation between the diet, and cognitive impairment, and 5 studies found no association between the diet and cognitive function [28]. 
Table 1: Individual food items indicating beneficial effects on dementia.

\begin{tabular}{|c|c|c|}
\hline Foods & Benefits & Reference \\
\hline Fish & Inversely linked & $14,1,15,18,21$ \\
\hline Wine & Inversely linked & $13,14,15$ \\
\hline Red wine & Inversely linked & $14,15,23,28$ \\
\hline Olive oil & Inversely linked & $13,14,15,18$ \\
\hline Fruits & Inversely linked & $13,14,15,18$ \\
\hline Vegetables & Inversely linked & 1 \\
\hline Nuts & Inversely linked & 13,25 \\
\hline Coffee & Inversely linked & $13,26,27$ \\
\hline Green tea & Positively linked Not associated & 18,28 \\
\hline
\end{tabular}

\section{Medical Rice as Wole Grain For Dementia}

Medical rice is rich in functional ingredients; dietary fibers, GABA, selenium and magnesium phenolic acids and flavonoids, anthocyanins, pro-anthocyanidins, tocopherols, tocotrienols, $\gamma$-oryzanol, and phytic acid that provide a good balance of the bacterial flora and of the immune system, if consumed as whole grains [10,29-32]. Experimental study by Mamiya et al, 2013 suggested that continuous feeding of food pellets containing pre-germinated brown rice attenuates the Aß25-35-induced impairments of cognitive function, and inhibited increases in lipid peroxidation in the hippocampus related to cognition [10]. Whole grain is also an important ingredient of MIND diet (MediterraneanDASH Intervention for Neurodegenerative Delay,2015), incorporates many elements of the above-mentioned diets for neuroprotection [12]. Catechin flavonoids rich in tea and cocoa can reduce cognitive decline and the levels of beta-amyloid in a mouse model of Alzheimer's disease. Improvement in cognitive function is not always accompanied by a change in pathological features, so it is not known whether flavonoids have a direct impact on betaamyloid $[13,26,27]$. There is evidence that components of the germ layer and bran of Brown rice help maintain good health and prevent dementia [29-31]. Since the germ layer and bran absorb very less water and are very hard and difficult to cook, they are often removed during processing. To solve these problems, in this study, we tried to use a high-pressure technique to solubilize it. High pressure treatment increased the water absorbency of brown rice without nutrient loss. It also ameliorated cognitive dysfunction and reduced the levels of amyloid- $\beta$, which is a major protein responsible for Alzheimer's disease, in the brain. In this experiment the authors have analyzed the benefits of brown rice (BR, Oryza sativa L.) (Table 2) for the prevention of dementia and other dementias because it is widely cultivated and consumed in Asia and other regions. Brown rice is often processed to remove the germ layer and bran because they are very hard, difficult to cook, and poorly digested. Interestingly, these regions of rice contain many ingredients that can be used to prevent dementias.

Table 2: Nutrient content of brown rice and white rice.

\begin{tabular}{|c|c|c|}
\hline Nutrient Proximates & Brown Rice & White Rice \\
\hline Energy, K Cal & 82 & 1.42 \\
\hline Protein, g & 1.83 & 0.15 \\
\hline Total fat, g & 0.65 & 14.84 \\
\hline Carbohydrate, g & 17.05 & 0.2 \\
\hline Fiber, g & 1.1 & 0.03 \\
\hline Sugars, g & 0.16 & 0.04 \\
\hline Fatty acids, saturated, g & 0.17 & 0.63 \\
\hline Iron, $m g$ & 0.37 & \\
\hline Flavonoids, g & & \\
\hline Calcium, $\mathrm{mg}$ & 2.0 & 5.0 \\
\hline
\end{tabular}

Ferulic acid has antioxidant potential and it is neuroprotective effects and inhibits amyloid $\beta(A \beta)$ production and aggregation [32]. They also include vitamin B6, vitamin B12 and folic acid, which are essential for methionine metabolism; the deficiency of them leads to an increase in blood homocysteine levels, increasing the risk of vascular diseases and dementia. The levels of gamma-aminobutyric acid (GABA), which acts as an excitatory neurotransmitter in the brain and plays a crucial role in regulating nerve excitation, are reduced in the brains of patients with Alzeimers disease than in the brains of healthy adults [32]. The Indian Lifestyle and Heart study reported that the prevalence of cognitive decline and dementia was $2.95 \%(n=59)$ associated with diet score below 6 out of $1-10$, 
indicating increased intake of Western dietary pattern and lower Mediterranean diet pattern. There is potential epidemiological evidence of a strong correlation among diet, lifestyle factors and the onset and consolidation of dementia and Alzheimer's disease [28].

It has been demonstrated that dementia, diabetes, obesity, insulin resistance, and cardiovascular disease are strongly interconnected pathologies. Preventive strategies and nutritional interventions seem to be promising approaches to delay neurocognitive decline and reduce the risk of dementia and other non-psychiatric co-morbidities. In this regard, healthy dietary patterns, characterized by high intake of plant-based foods, probiotics, antioxidants, soy beans, nuts, and omega-3 polyunsaturated fatty acids, and a low intake of saturated fats, animal-derived proteins, and refined sugars, have been shown to decrease the risk of neurocognitive impairments and eventually the onset of dementia. In brief, the ageing population is accompanied by increased rates of cognitive decline and dementia, which have profound impact on an individual's health and quality of life, as well as on the life of their caregivers. the beneficial effects of Mediterranean diet are associated with overall diet composition, rather than single nutrient supplementations, for the prevention or the delay of Alzheimer's disease and dementia. The Mediterranean diet has been known to aid in reducing the risk of CVDs, cancer and diabetes. The highest beneficial components of Mediterranean style diets are vegetables and whole grains, because they are rich in polyphenolics and flavonoids. Medical rice is a whole grain which is rich in GABA, selenium and magnesium phenolic acids and flavonoids; anthocyanins, pro-anthocyanidins, tocopherols, tocotrienols, $\gamma$-oryzanol which have potential antioxidant effects [29]. Flavonoids have been recently linked to better cognitive function in the elderly population. However, it should be noted that the majority of findings come from epidemiologic studies that provide evidence for a correlation between the Mediterranean diets and cognition but not for a cause and effect relation. More controlled trials are required to establish a causational relation.

\section{References}

1. Singh RB, Rastogi SS, Verma, R Laxmi, B Singh R (1992) Randomized, controlled trial of cardio protective diet in patients with acute myocardial infarction: results of one year follow up. BMJ 304(6833): 1015-1019.

2. De Lorgeril M, Renaud S, Mamelle N, Salen P, Martin JL, et al. (1994) Mediterranean alpha-linolenic acid-rich diet in secondary prevention of coronary heart disease. Lancet 343(8911):1454-1459.

3. Singh RB, Dubnov G, Niaz MA, Ghosh S, Singh R, et al. (2002) Effect of an Indo-Mediterranean diet on progression of coronary disease in high risk patients:a randomized single blind trial. Lancet 360(9344): 1455-1461.

4. Esposito K, Marfella R, Ciotola M, DiPalo C, Giugliano G, et al. (2004) Effect of a Mediterranean-style diet on endothelial dysfunction and markers of vascular inflammation in the metabolic syndrome: a randomized trial. JAMA 292(12): 1440-1446.

5. Petersson SD, Philippou E (2016) Mediterranean Diet, Cognitive Function, and Dementia: A Systematic Review of the Evidence. Adv Nutr 7(5): 889-904.
6. Aridi YS, Walker JL, Wright ORL (2017) The Association between the Mediterranean Dietary Pattern and Cognitive Health: A Systematic Review. Nutrients: 9(7).

7. Martínez Lapiscina EH, Clavero P, Toledo E, Estruch R, Salas Salvadó J et al. (2013) Mediterranean diet improves cognition: the PREDIMEDNAVARRA randomised trial. J Neurol Neurosurg Psychiatry. 84(12):13181325.

8. Oannis Bakoyiannis, Afrodite Daskalopoulou, Vasilios Pergialiotis, Despina Perrea (2019) Phytochemicals and cognitive health: Are flavonoids doing the trick. Biomedicine \& Pharmacotherapy 109: 14881497.

9. Morris MC (2016) Nutrition and risk of dementia: overview and methodological issues. Ann N Y Acad Sci 1367(1): 31-37.

10. Mamiya T, Ukai M, Morikawa K, Kise M (2013) Intake of food pellets containing pre-germinated brown rice alleviates cognitive deficits caused by $\beta$-amyloid peptide25-35 in mice: Implication of lipid peroxidation. J Rice Res 1:116.

11. Reichelt AC, Stoeckel LE, Reagan LP, Winstanley CA, Page KA (2018) Prospective Associations between Single Foods, Alzheimer's Dementia and Memory Decline in the Elderly. Physiology \& behavior. Mar 1; 192: 118-126

12. Morris MC, Tangney CC, Wang Y, et al. (2015) MIND diet slows cognitive decline with aging. Alzheimers Dement 11(9):1015-1022.

13. 13Hu N, Yu JT, Tan L, Wang YL, Sun L, et al. (2013) Nutrition and the risk of Alzheimer's disease. Biomed Res Int :524820.

14. Solfrizzi V, Panza F, Frisardi V, Seripa D, Logroscino G, et al. (2011) Diet and Alzheimer's disease risk factors or prevention: The current evidence. Expert Rev. Neurother 11(5): 677-708.

15. Parletta N, Milte CM, Meyer BJ (2013) Nutritional modulation of cognitive function and mental health. J Nutr Biochem 24(5): 725-743.

16. Mi W, Van Wijk N, Cansev M, Sijben JW, Kamphuis PJ (2013) Nutritional approaches in the risk reduction and management of Alzheimer's disease. Nutrition 29(9): 1080-1089.

17. Swaminathan A, Jicha GA (2014) Nutrition and prevention of Alzheimer's dementia. Front. Aging Neurosci 6: 282.

18. Otaegui Arrazola A, Amiano P, Elbusto A, Urdaneta E, Martínez Lage P (2014) Diet, cognition, and Alzheimer's disease: Food for thought. Eur J Nutr 53(1):1-23.

19. Jacobs DR, Steffen LM (2003) Nutrients, foods, and dietary patterns as exposures in research: A framework for food synergy. Am J Clin Nutr 78(3): 508-513.

20. Jacobs DR, Tapsell LC (2007) Food, not nutrients, is the fundamental unit in nutrition. Nutr Rev 65(10): 439-450.

21. Cederholm T (2017) Fish consumption and omega-3 fatty acid supplementation for prevention or treatment of cognitive decline, dementia or Alzheimer's disease in older adults-Any news? Curr. Opin Clin Nutr Metab Care.

22. Arntzen KA, Schirmer H, Wilsgaard T, Mathiesen EB (2010) Moderate wine consumption is associated with better cognitive test results: A 7 year follow up of 5033 subjects in the Tromso Study. Acta Neurol Scand 190: 23-29.

23. Berr C, Portet F, Carriere I, Akbaraly TN, Feart C, et al. (2009) Olive oil and cognition: Results from the three-city study. Dement Geriatr. Cogn. Disord 28(4): 357-364.

24. Loef M, Walach H (2012) Fruit, vegetables and prevention of cognitive decline or dementia: A systematic review of cohort studies. J. Nutr. Health Aging 16(7): 626-630.

25. Liu QP, Wu YF, Cheng HY, Xia T, Ding H et al. (2016) Habitual coffee consumption and risk of cognitive decline/dementia: A systematic 
review and meta-analysis of prospective cohort studies. Nutrition 32(6): 628-636.

26. Mandel SA, Youdim MB (2012) In the rush for green gold: Can green tea delay age-progressive brain neurodegeneration? Recent Pat. CNS Drug Discov 7(3): 205-217.

27. Lim HJ, Shim SB, Jee SW, Lee SH, Lim CJ, et al. (2013) Hwang D.Y. Green tea catechin leads to global improvement among Alzheimer's diseaserelated phenotypes in NSE/hAPP-C105 Tg mice. J Nutr Biochem 24(7): 1302-1313.

28. Pistollato F, Iglesias RC, Ruiz R, Aparicio S, Crespo J, et al. (2018) Nutritional patterns associated with the maintenance of neurocognitive functions and the risk of dementia and Alzheimer's disease: A focus on human studies. Pharmacol Res 131: 32-43.

ISSN: 2574-1241

DOI: 10.26717.BJSTR.2019.14.002595

Viliam Mojto. Biomed J Sci \& Tech Res

(C) This work is licensed under Creative Commons Attribution 4.0 License

Submission Link: https://biomedres.us/submit-manuscript.php
29. Watanabe S, Endo K, Nakajou M, Takei N, Beppu S (2018) Low protein rice: medical Rice for chronic kidney Disease. In: RB Singh, Toru Takahashi, RR Watson. Role of Functional Food in Global Health, Elsevier, USA.

30. Watanabe S, Hirakawa A, Nishijima C, Ohtsubo K, Nakamura K, et al (2016) Food as medicine: the new concept of "medical rice". Adv Food Technol Nutr Sci Open J 2(2): 38-50.

31. Watanabe S (2018) Population-based strategy for preventing diabetes and its complications. Diabetes Res Open J 4(1): 1-4.

32. Okuda M, Fujita Y, Katsube T (2018) Highly water pressurized brown rice improves cognitive dysfunction in senescence-accelerated mouse prone 8 and reduces amyloid beta in the brain. BMC Complement Altern Med 18(1): 110.

\begin{tabular}{ll} 
BIOMEDICAL & Assets of Publishing with us \\
RESEARCHES & - Global archiving of articles \\
& - Immediate, unrestricted online access \\
\hline
\end{tabular}

